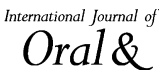 Maxillofacial Surgery \\ Research Paper Reconstructive Surgery}

\section{Digital surface scanning in flap perfusion}

J.T. Deferm, F. Baan, R. Schreurs, R. Willaert, T. Maal, G. Meijer: Digital surface scanning in flap perfusion. Int. J. Oral Maxillofac. Surg. 2019; xxx: xxx-xxx. (C) 2020 International Association of Oral and Maxillofacial Surgeons. Published by Elsevier Ltd. All rights reserved.

Abstract. Monitoring vascular perfusion of transferred tissue is essential in reconstructive surgery to recognize early flap failure. The aim of this study was to evaluate the ability of a digital surface scanner to detect vascular perfusion disorders through the monitoring of skin colour changes. A total of 160 surface scans of the forearm skin were performed with a TRIOS 3D scanner. Vascular compromise was simulated at different time-points by intermittent occlusion of the blood supply to the forearm skin (first the arterial blood supply and then the venous blood supply). Skin colour changes were examined according to the hue, saturation, and value colour scale. Colour differences were analysed with a paired $t$-test. Significant differences were observed between the colour of the normal skin and that of the vascular compromised skin $(P<0.01)$. The surface scanner could distinguish between arterial occlusion and venous congestion $(P<0.01)$. A digital surface scan is an objective, non-invasive tool to detect early vascular perfusion disorders of the skin.

\author{
J. T. Deferm ${ }^{1}$, F. Baan ${ }^{2,3}$, \\ R. Schreurs ${ }^{3,4}$, R. Willaert ${ }^{5,6}$, \\ T. Maal ${ }^{1,3}$, G. Meijer ${ }^{1,7}$
}

${ }^{1}$ Department of Oral and Maxillofacial Surgery, Radboud University Nijmegen Medical Centre, Nijmegen, The Netherlands; ${ }^{2}$ Department of Orthodontics and Craniofacial Biology, Radboud University Nijmegen Medical Centre, Nijmegen, The Netherlands; ${ }^{3}$ Radboudumc 3D Lab, Radboud University Nijmegen Medical Centre, Nijmegen, The Netherlands; ${ }^{4}$ Department of Oral and Maxillofacial Surgery, Amsterdam UMC Location AMC and Academic Center for Dentistry Amsterdam (ACTA), University of Amsterdam, Amsterdam, The Netherlands; ${ }^{5}$ Department of Oral and Maxillofacial Surgery, University Hospitals Leuven, Leuven, Belgium; ${ }^{6}$ Department of Head and Neck Surgery, Ghent University Hospital, Ghent, Belgium; ${ }^{7}$ Department of Implantology and Periodontology, Radboud University Nijmegen Medical Centre, Nijmegen, The Netherlands

Key words: reconstructive surgical procedures; free tissue flap; three-dimensional image; perfusion; ischemia; venous thrombosis; colour.

Accepted for publication 21 May 2020
Microvascular free flaps are used for the repair of various tissue defects. Flap failure occurs predominantly in the first 24 hours postoperative, due to vascular compromise of the transferred tissue ${ }^{1}$. Causes of vascular compromise include both arterial pathologies, such as arterial thrombosis, vasospasm, and anastomotic failure, and venous pathologies, such as extrinsic venous compression and venous thrombosis ${ }^{1}$. An assessment of vascular tissue perfusion and early detection of flap failure are essential to achieve the best chance of successful surgical salvage and flap survival ${ }^{2}$. Monitoring of the microvascular flap is paramount to the early recognition of failure. Clinical examination of the colour, capillary refill, tissue turgor, oedema, and bleeding characteristics of the free flap is still considered the gold standard for a majority of microvascular surgeons ${ }^{1,3-5}$. A limitation of this approach is human error in recognizing changes in these parameters ${ }^{3}$. Current technology can be used to optimize the clinical monitoring techniques, in order to analyse and detect early vascular compromise after free flap reconstruction. The ideal monitoring technique needs to be harmless to the patient, rapid, accurate, reliable, and applicable to all flap types, as well as inexpensive, easy to perform, and easily interpretable by the nursing staff $^{3,6}$.
As the colour of the skin is considered to be the most predictable clinical indicator of the vascular perfusion, objective quantification of skin colour changes could be useful to examine the flap viability ${ }^{7}$. The aim of this study was to determine whether a digital surface scanner can recognize early skin colour alterations as a result of a vascular compromise.

\section{Materials and methods}

A group of 10 healthy Caucasian volunteers (six male, mean age of $29 \pm 5$ years) were recruited prospectively. Impaired vascular perfusion was simulated at different time-points by intermittent 
occlusion of the blood supply to the forearm skin.

Inclusion criteria were (1) minimum age of 18 years, (2) non-smoker with no medical history of vascular disease, and (3) systolic blood pressure between 100 $\mathrm{mmHg}$ and $140 \mathrm{mmHg}$, and diastolic blood pressure between $60 \mathrm{mmHg}$ and $80 \mathrm{mmHg}$. Various Fitzpatrick types (type I to IV) were included. Volunteers with a negative Allen manoeuvre, Sudeck atrophy, or who had undergone previous surgical interventions involving the forearm were excluded. The study adhered to the tenets of the Declaration of Helsinki. Institutional review board approval and informed consent were obtained (2016-2690).

\section{Data acquisition}

Multiple surface scans of the participants' forearm skin were acquired with a TRIOS 3D scanner (3Shape, Copenhagen, Denmark). One observer performed all of the surface scans according to the scanning protocol provided by the manufacturer $^{8}$. To simulate the radial forearm flap design, a square of $4 \times 5 \mathrm{~cm}$ centred over the radial artery was drawn to define the region of interest for scanning.

A baseline scan was obtained at the beginning to document the normal physiological state. Subsequently, the cuff of a standard manual mercury sphygmomanometer was applied to the upper arm to alter the vascular perfusion of the forearm. To simulate arterial occlusion, the participant was asked to hold the arm in $180^{\circ}$ anteflexion for 30 seconds to deplete the superficial venous blood system. The cuff was then inflated to $220 \mathrm{mmHg}$. The forearm skin was scanned after 1 and 10 minutes of arterial occlusion (respectively AO $1^{\prime}$ and AO $10^{\prime}$ ). After this 10-minute scan, the cuff was deflated to restore normal vascular flow, and the forearm skin was scanned 1 minute after deflation to measure revascularization after arterial occlusion (RAO). After a break of approximately 25 minutes, venous congestion was simulated by inflating the cuff to $40 \mathrm{mmHg}$. As this pressure is higher than central venous pressure and lower than diastolic pressure, the arterial blood supply will not be compromised. Surface scans of the forearm skin were acquired after 1 and 10 minutes of venous congestion (respectively $\mathrm{VC} 1^{\prime}$ and $\mathrm{VC} 10^{\prime}$ ). To return venous circulation, the cuff was deflated; 1 minute after deflation, the forearm skin was scanned to measure revascularization after venous congestion (RVC). Finally, 10 minutes after the last scan, a control surface scan was obtained with the cuff removed and the forearm in neutral position. Identical procedures were performed on the contralateral forearm.

\section{Measurements}

To investigate skin colour changes, all surface scans were imported into 3DS Max v2016 software (Autodesk Inc., San Rafael, CA, USA). After cropping the irrelevant areas towards the region of interest, the surface colour was extracted in hue $(\mathrm{H})$, saturation $(\mathrm{S})$, and value (V) format (Fig. 1). The HSV colour scale is a cylindrical geometry whereby 'hue', ranging from 0 to $360^{\circ}$, is formed in a radial slice and can be seen as the basic aspect of a colour. The central axis of neutral colours ranges from black at the bottom to white at the top, representing the 'value' (between 0 and 100\%) as the brightness of a colour. The 'saturation' refers to the intensity or purity of a colour (between 0 and $100 \%)^{9}$.

\section{Statistical analysis}

All data were analysed using IBM SPSS Statistics version 22 (IBM Corp., Armonk, NY, USA). Mean and standard deviation (SD) values were calculated. A paired $t$ test (two-sided) was used to compare the scan results during the different vascular stages and at the different time-points. A
$P$-value of $<0.01$ was set as the level of statistical significance.

\section{Results}

A total of 160 digital surface scans of the forearm skin were obtained. An example of the colour changes during the different stages of vascular compromise are depicted in Fig. 2.

The mean results of the skin colour measurements for each stage and at each time point are reported in Table 1. The temporal evolution of skin colour changes during vascular compromise is shown in Fig. 3. The HSV curves all showed a sinusoidal shape in which the measurements obtained during arterial occlusion displayed an opposite tendency compared to those obtained during the stage of venous congestion. The curve amplitude was most pronounced for $\mathrm{H}$. Transitions of the $S$ curve were more levelled and progressed inversely compared to the $\mathrm{V}$ and $\mathrm{H}$ curves. The colour measurements at the control stage demonstrated a return towards the baseline for $\mathrm{H}, \mathrm{S}$, and $\mathrm{V}$. The skin colour changes differed significantly between the baseline measurement and immediate arterial occlusion and venous congestion for $\mathrm{H}, \mathrm{S}$, and $\mathrm{V}(P<0.01$ for baseline vs. AO $1^{\prime}$ and for baseline vs. VC $\left.1^{\prime}\right)$. Significant skin colour changes were observed as venous congestion continued $(P<0.01$ $(\mathrm{H})$ for $\mathrm{VC} 1^{\prime}$ vs. $\left.\mathrm{VC} 10^{\prime}\right)$. In contrast, no significant colour changes were seen

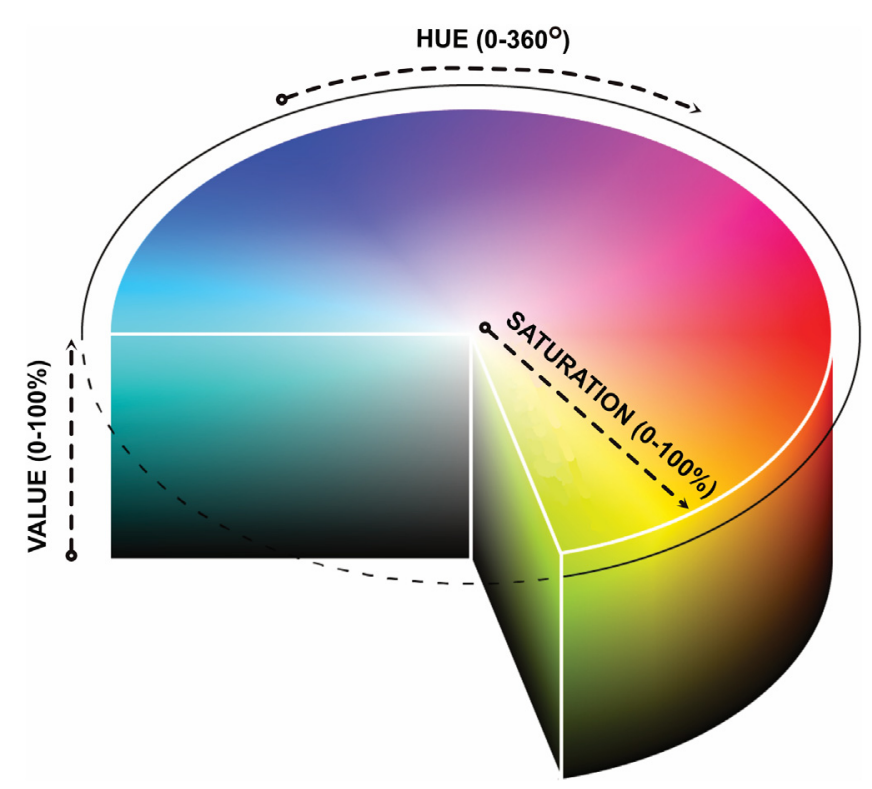

Fig. 1. A cylindrical colour model defining hue $(\mathrm{H})$, saturation $(\mathrm{S})$, and value (V). 'Hue', ranging from 0 to $360^{\circ}$, can be seen as the basic aspect of a colour. The colour brightness is determined by the height of the cylinder as 'value'. 'Saturation' refers to the intensity or purity of a colour and varies from the central axis towards the cylinder periphery. 


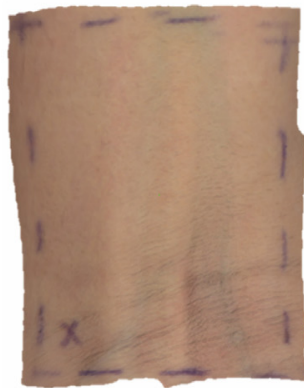

BASELINE

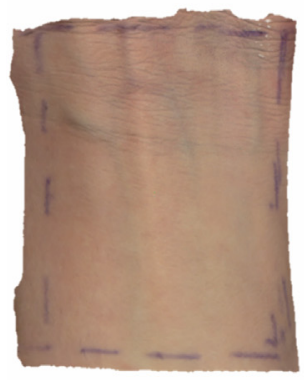

BASELINE

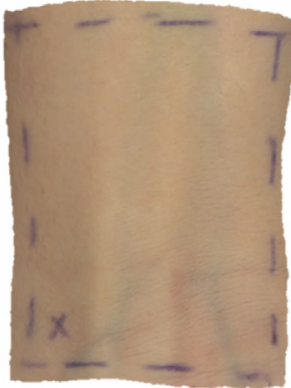

AO 1'

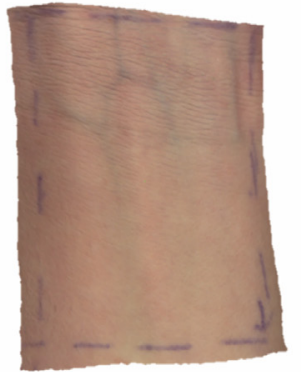

VC 1'

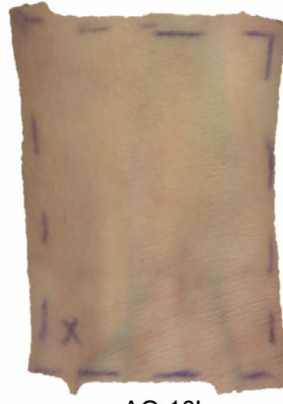

AO 10'

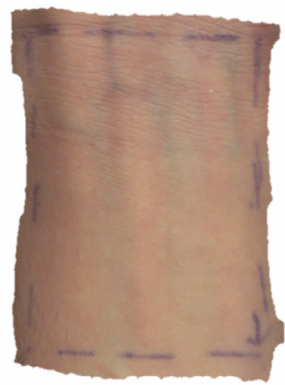

VC $10^{\prime}$

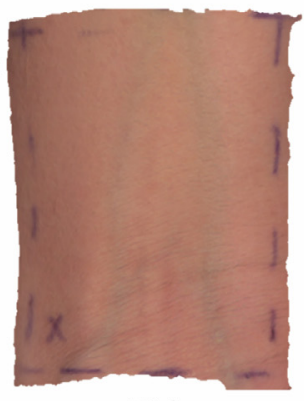

RAO

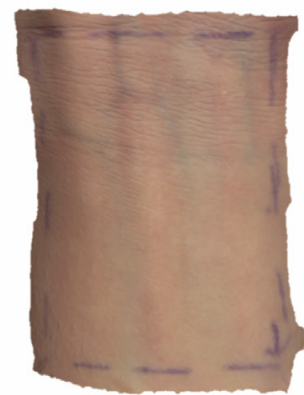

RVC

Fig. 2. Successive digital surface scans of the forearm skin during different stages of simulated vascular compromise. The region of interest was marked on the forearm skin before scanning (interrupted line). Skin colour changes are illustrated during arterial occlusion (AO) and venous congestion (VC) after 1 minute $\left(1^{\prime}\right)$ and 10 minutes $\left(10^{\prime}\right)$. Immediate recovery of the normal blood flow is shown at the end (revascularization after arterial occlusion (RAO) and after venous congestion (RVC)). Although the subjective colour changes could be very subtle, digital analysis of the colour features was able to detect objective and significant differences.

Table 1. Skin colour measurements at the different stages during vascular compromise; mean \pm standard deviation values.

\begin{tabular}{llll}
\hline & Hue $\left(^{\circ}\right)$ & Saturation $(\%)$ & Value $(\%)$ \\
\hline Baseline & $20 \pm 3$ & $34 \pm 5$ & $71 \pm 4$ \\
AO 1 & \\
AO $10^{\prime}$ & $30 \pm 3^{\mathrm{a}, \mathrm{c}}$ & $32 \pm 5^{\mathrm{a}, \mathrm{c}}$ & $74 \pm 3^{\mathrm{a}, \mathrm{c}}$ \\
RAO & $30 \pm 3^{\mathrm{a}}$ & $31 \pm 5^{\mathrm{a}, \mathrm{b}}$ & $73 \pm 3^{\mathrm{a}, \mathrm{b}}$ \\
VC 1 & $11 \pm 3^{\mathrm{b}}$ & $41 \pm 4^{\mathrm{b}}$ & $72 \pm 3^{\mathrm{b}}$ \\
VC 10 & $15 \pm 3^{\mathrm{a}}$ & $35 \pm 4^{\mathrm{a}}$ & $68 \pm 3^{\mathrm{a}}$ \\
RVC & $12 \pm 3^{\mathrm{a}, \mathrm{b}}$ & $35 \pm 4^{\mathrm{a}}$ & $65 \pm 3^{\mathrm{a}, \mathrm{b}}$ \\
Control & $16 \pm 3^{\mathrm{b}}$ & $35 \pm 4$ & $68 \pm 3^{\mathrm{b}}$ \\
\hline
\end{tabular}

$1^{\prime}$ : 1 minute; 10': 10 minutes; $\mathrm{AO}$, arterial occlusion; RAO, revascularization after arterial occlusion; RVC, revascularization after venous congestion; VC, venous congestion.

${ }^{\text {a }} P<0.01$ compared to the baseline scan.

${ }^{\mathrm{b}} P<0.01$ compared to the previous stage.

${ }^{\mathrm{c}} P<0.01$ compared to venous congestion after 1 minute. $P$-values were obtained with a paired two-sided $t$-test.

during prolonged arterial occlusion $\left(P=0.67(\mathrm{H})\right.$ for $\mathrm{AO} 1^{\prime}$ vs. AO $\left.10^{\prime}\right)$. Revascularization after arterial occlusion and venous congestion illustrated a significant colour shift for both $\mathrm{H}$ and $\mathrm{V}$ $\left(P<0.01\right.$ for RAO vs. AO $10^{\prime}$ and for RVC vs. VC $10^{\prime}$ ). A significant difference in skin colour was seen for arterial occlusion and venous congestion at the same time-point $\left(P<0.01(\mathrm{H})\right.$ for AO $1^{\prime}$ vs. VC $\left.1^{\prime}\right)$. Finally, the control scan at the end of the experiment and the baseline scan differed significantly for $\mathrm{H}(P<0.01$ for baseline vs. control), but not for $\mathrm{S}$ or $\mathrm{V}$.

\section{Discussion}

Survival of a free flap reconstruction depends predominantly on the vascular tissue perfusion. Ischemia due to occlusion of the supplying artery or congestion caused by obstruction of the venous outflow should be recognized as soon as possible to enhance the success rate of salvage surgery ${ }^{1}$. Clinical observation remains the gold standard to assess the vascularization of the transferred tissue, but it is crucial to acknowledge that this is a subjective evaluation, highly dependent on the observer's experience.
Various monitoring techniques have been developed to assist flap surveillance in a more objective way. Techniques monitoring vascular flow include hand-held Doppler ultrasonography, laser Doppler flowmetry, colour duplex ultrasonography, fluorescence angiography, implantable Doppler, and implantable flow coupler $^{1,4}$. Doppler ultrasonography and laser Doppler flowmetry are both excellent non-invasive tools with high positive and negative predictive values. Unfortunately, they are not able to differentiate between arterial and venous compromise. Fluorescent angiography and colour duplex ultrasonography are great adjunctive techniques in preoperative planning and in the intra- and postoperative quantification of perfusion. Nevertheless, they are not recommended as primary flap monitoring tools given the non-continuous nature of use. Implantable Doppler and flow coupler can directly monitor the arterial and venous flow. Disadvantages of these approaches include the invasiveness of attachment of the probe around the vascular anastomosis. Techniques monitoring tissue metabolism and ischemia include near-infrared spectroscopy, which uses dyes such as indocyanine green, and microdialysis to determine the concentrations of glucose, lactate, and pyruvate in 


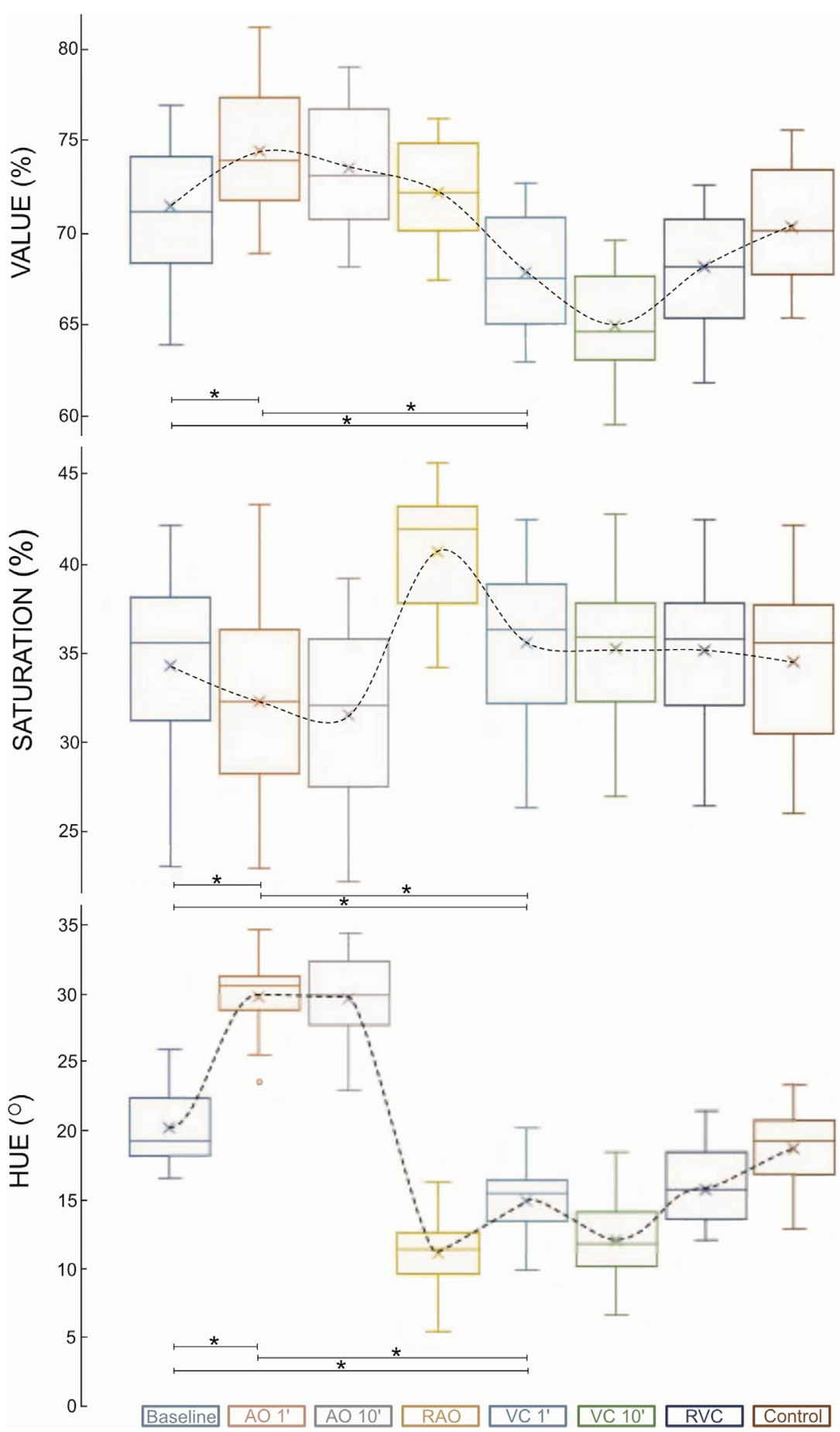

Fig. 3. Change in colour features during the consecutive stages of compromised vascularity of the skin. The boxplots represent the colour measurements at each stage of impaired vascularity. Evolution of the means (x) is shown for hue $(\mathrm{H})$, saturation $(\mathrm{S})$, and value $(\mathrm{V})$ (dotted line). Statistically significant differences $(*)$ were found between the baseline surface scan and 1 minute of arterial occlusion (baseline vs $\mathrm{AO} 1^{\prime}$ ) and 1 minute of venous congestion (baseline vs $\mathrm{VC} 1^{\prime}$ ), as well as between arterial occlusion and venous congestion (AO $1^{\prime}$ vs $\mathrm{VC} 1^{\prime}$ ). Measurement differences between the different stages were most obvious for $\mathrm{H}$, which represents the basic feature of a colour.

the flap as surrogate markers of tissue perfusion $^{4,5,10}$. Both methods show great potential for the early detection of flap failure, however they are invasive, expensive, and require some user experience for an accurate interpretation.

At this time, there is no evidence of any particular technique being superior for the early detection of vascular failure of the reconstructed tissue ${ }^{1,4}$. Optimizing the current clinical monitoring protocols with advanced technological devices could be beneficial. Since the colour of a free flap is a crucial clinical predictor of the viability of the flap, this study investigated whether the assessment of skin colour, obtained by a digital surface scanner, is able to detect early impairment of vascular tissue perfusion. An intraoral scanner was chosen for three reasons. This handheld device can provide a fast and accurate image of the soft tissue colour ${ }^{10}$. Furthermore, the scanning procedure with the TRIOS 3D scanner is non-invasive and easy to perform, even for an inexperienced observer $^{10-12}$. Previous studies have illustrated that scanning of the soft tissues inside the oral cavity is feasible, which makes this device more suitable for colour assessment in difficult to reach areas with changing ambient light ${ }^{13,14}$.

The results of this study emphasize that colour assessment by digital scanning of the skin surface can detect impaired vascular tissue perfusion. Simulations of arterial occlusion and of venous congestion provoked alterations of the skin colour that were significantly different to the baseline condition. The change in skin colour due to vascular compromise could already be identified after 1 minute. This indicates that a digital surface scanner is able to discern vascular compromise at an early stage, which could be a great advantage for the rapid detection of a potential free flap failure. Furthermore, the scanner can distinguish arterial ischemia from venous congestion. This could be useful in identifying the source of vascular impairment and determining the optimal treatment.

As seen in this study, hue appears to be the most obvious colour aspect when evaluating vascular changes. In the case of an arterial occlusion, the forearm skin colour will lose its red appearance and become more yellow and pale. This correlates with the study findings, as hue increased from $20^{\circ}$ towards $30^{\circ}$, indicating a shift from red-orange towards the yellow spectrum. During venous congestion, expressed as a darker red or purple aspect of the forearm skin colour, the opposite trend is observed; the scanner detected a decrease in hue from $20^{\circ}$ to $12^{\circ}$.

Recovery of the vascular perfusion resulted in significant skin colour changes that were most apparent when revascularization occurred after arterial occlusion. Restoration of the circulation after venous congestion is less obvious by clinical observation, but still a significant difference in hue was detected in the present study 
measurements, illustrating the benefit of the surface scan to supplement the subjective clinical assessment. While value and saturation had normalized almost entirely at the time of the control scan, hue recovered more slowly and differed significantly when compared to the baseline scan. It is likely that the measurements obtained at the time of the control scan were not completely representative yet, due to the relatively short recovery time (10 minutes); this could have been insufficient to completely reoxygenate the tissues to a normal level.

There are several limitations of the study. Further investigation is required to elucidate how the colour characteristics of the soft tissue will evolve during a surgical procedure. The surgical trauma will inevitably cause vascular and skin colour changes due to tissue reaction. Likewise, the variance in blood pressure or changes in the haemoglobin status of the patient could affect measurement outcomes. The scan results provide an objective measurement suggesting a vascular skin perfusion disorder, but there are no absolute threshold values indicating ischemia or congestion. As skin colour values at baseline are different in every patient, the evolution of relative changes between measurement stages is most informative, and unusual colour changes should be considered as a warning sign. The study was performed on Caucasian participants and it would be interesting to investigate the skin colour changes in other ethnic groups as well. Only low-grade skin phototypes (grades I to IV) were included, and investigations should be performed to determine whether these findings also apply to the higher Fitzpatrick skin types. Finally, this study was only performed with a simulation of vascular compromise in healthy participants. Thus further research is required to allow clinical implementation of this monitoring technique after tissue transfer in patients with co-morbidities.

This study is novel in attempting to use a digital surface scanner to discern vascular tissue perfusion. Although the results show it is feasible to detect skin colour changes during various stages of impaired tissue perfusion, more studies are needed to elucidate the clinical advantages of this technique during the monitoring of a microvascular free flap.

\section{Funding}

This study did not receive any funding.

\section{Competing interests}

There are no competing interests to disclose.

\section{Ethical approval}

The study adhered to the tenets of the Declaration of Helsinki. Institutional review board approval was obtained (2016-2690).

\section{Patient consent}

Not required.

\section{References}

1. Karinja SJ, Lee BT. Advances in flap monitoring and impact of enhanced recovery protocols. J Surg Oncol 2018;118:758-67.

2. Brennan PA, Brands MT, Gush R, Alam P. Laser-speckle imaging to measure tissue perfusion in free flaps in oral and maxillofacial surgery: a potentially exciting and easy to use monitoring method. $\mathrm{Br} J$ Oral Maxillofac Surg 2018;56:556-8.

3. Urken ML, Weinberg H, Vickery C, Buchbinder D, Biller HF. Free flap design in head and neck reconstruction to achieve an external segment for monitoring. Arch Otolaryngol Neck Surg 1989;115:1447-53.

4. Kääriäinen M, Halme E, Laranne J. Modern postoperative monitoring of free flaps. Curr Opin Otolaryngol Head Neck Surg 2018;26: 248-53.

5. Jyranki J, Suominen S, Vuola J, Back L. Microdialysis in clinical practice: monitoring intraoral free flaps. Ann Plast Surg 2006;56:387-93.

6. Creech B, Miller S. Evaluation of circulation in skin flaps. In: Grabb WC, Myers MB, editors. Skin flaps. Boston: Little, Brown and Company; 1975.

7. Van Genechten M, Rahmel B, Batstone MD. Red or white? Use of high colour-rendering index, light-emitting diodes in monitoring of free flaps of the head and neck. Br J Oral Maxillofac Surg 2015;53:765-6.

8. 3Shape instructions. https://www.3shape. $\mathrm{com} / \mathrm{en} / \mathrm{support-docs}$ [Accessibility verified September 6, 2019].

9. Li S, Guo G. The application of improved HSV color space model in image processing. Proceedings of the 2010 2nd International Conference on Future Computer and Communication ICFCC 2010. http://dx.doi.org/ 10.1109/ICFCC.2010.5497299. V2-10 V2-13.

10. Deferm JT, Schreurs R, Baan F, Bruggink R, Merkx MAW Xi T, Bergé SJ, Maal TJJ. Validation of 3D documentation of palatal soft tissue shape, color, and irregularity with intraoral scanning. Clin Oral Investig 2018;22:1303-9.

11. Kim J, Park JM, Kim M, Heo SJ, Shin IH, Kim M. Comparison of experience curves between two 3-dimensional intraoral scanners. J Prosthet Dent 2016;116:221-30.

12. Park HR, Park JM, Chun YS, Lee KN, Kim M. Changes in views on digital intraoral scanners among dental hygienists after training in digital impression taking. BMC Oral Health 2015;15:151.

13. Kattadiyil MT, Mursic Z, AlRumaih H, Goodacre CJ. Intraoral scanning of hard and soft tissues for partial removable dental prosthesis fabrication. $J$ Prosthet Dent 2014;112:444-8.

14. Gan N, Xiong Y, Jiao T. Accuracy of intraoral digital impressions for whole upper jaws, including full dentitions and palatal soft tissues. PLoS One 2016;11:e158800.

Address:

Julie T. Deferm

Department of Oral and Maxillofacial

Surgery

Geert Grooteplein 10

6525 GA Nijmegen

Gelderland

The Netherlands

Tel.: +312436145 50;

Fax: +31243541165

E-mail: julie.deferm@radboudumc.nl 